Supporting Information

\title{
Selective-Area Remote Epitaxy of ZnO Microrods using Multilayer-Monolayer-Patterned Graphene for Transferable and Flexible Device Fabrications
}

Junseok Jeong, ${ }^{\dagger, \ddagger}$ Dae Kwon Jin,,${ }^{\dagger,}$ Janghwan Cha,,$\$$ Bong Kyun Kang,,${ }^{\mathbb{f l}}$ Qingxiao Wang, ${ }^{\#}$ Joonghoon Choi, ${ }^{\dagger}{ }^{\dagger}$ Sang Wook Lee, ${ }^{\Delta}$ Vladimir Yu. Mikhailovskii, ${ }^{\top}$ Vladimir Neplokh, Nuño Amador-Mendez, ${ }^{\cap}$ Maria Tchernycheva, ${ }^{\cap}$ Woo Seok Yang, ${ }^{\mathbb{f l}}$ Jinkyoung Yoo, ${ }^{\perp}$ Moon J.

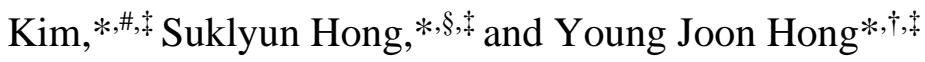

${ }^{\dagger}$ Department of Nanotechnology and Advanced Materials Engineering, ${ }^{\S}$ Department of Physics and Astronomy, ${ }^{\star}$ GRI-TPC International Research Center, Sejong University, Seoul 05006, Republic of Korea

"Nano Materials Research Center, Korea Electronics Technology Institute, Seongnam, Gyeonggi-do 13509, Republic Korea

\#Department of Materials Science \& Engineering, The University of Texas at Dallas, Richardson, TX 75080, the United States

${ }^{\Delta}$ Department of Physics, Ewha Womans University, Seoul 03760, Republic of Korea

『Saint Petersburg State University, 7/9 Universitetskaya Nab., 199034 St. Petersburg, Russia

Alferov University, Khlopina 8/3, 194021, St. Petersburg, Russia

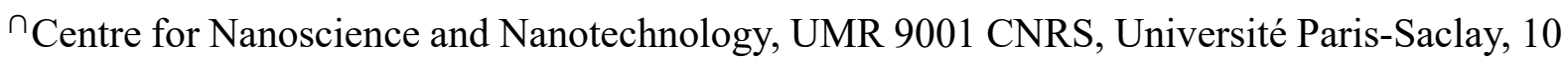
Boulevard Thomas Gobert, 91120 Palaiseau, France

${ }^{\perp}$ Center for Integrated Nanotechnologies, Los Alamos National Laboratory, Los Alamos, NM 87545, the United States

*Corresponding author: Y.J.H (E-mail: yjhong@sejong.ac.kr); S.H (E-mail: hong@sejong.ac.kr); M.J.K (E-mail: moonkim@utdallas.edu) 
(a)

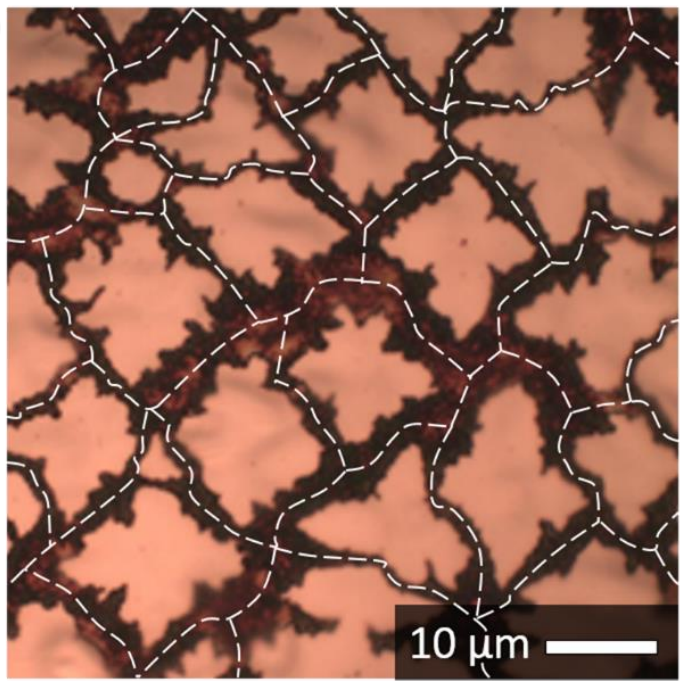

(b)

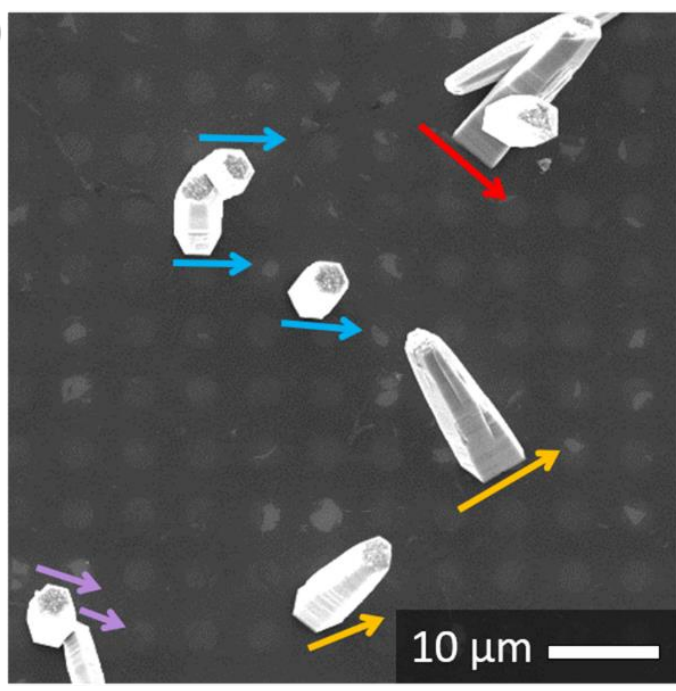

Figure S1. Impact of the underlying substrate in selective-area remote heteroepitaxy. (a) Optical microscopic image of single-layer graphene (SLG) before complete coalescence. White dashed lines are drawn to represent the domain boundary of SLG after lateral growth. (b) Planview FE-SEM image of $\mathrm{ZnO}$ MRs on intaglio-patterned graphene (IPG)-coated $\mathrm{SiO}_{2}$ substrate. The selective-area growth was failed using $\mathrm{SiO}_{2}$ wafer. Colored arrows indicate the hexagonal sidewall facet orientation of MRs. The adjacent MRs showed the same facet orientations, as marked with different color arrows. This implies that the crystallographic orientation of MRs was determined by the graphene of IPG. 


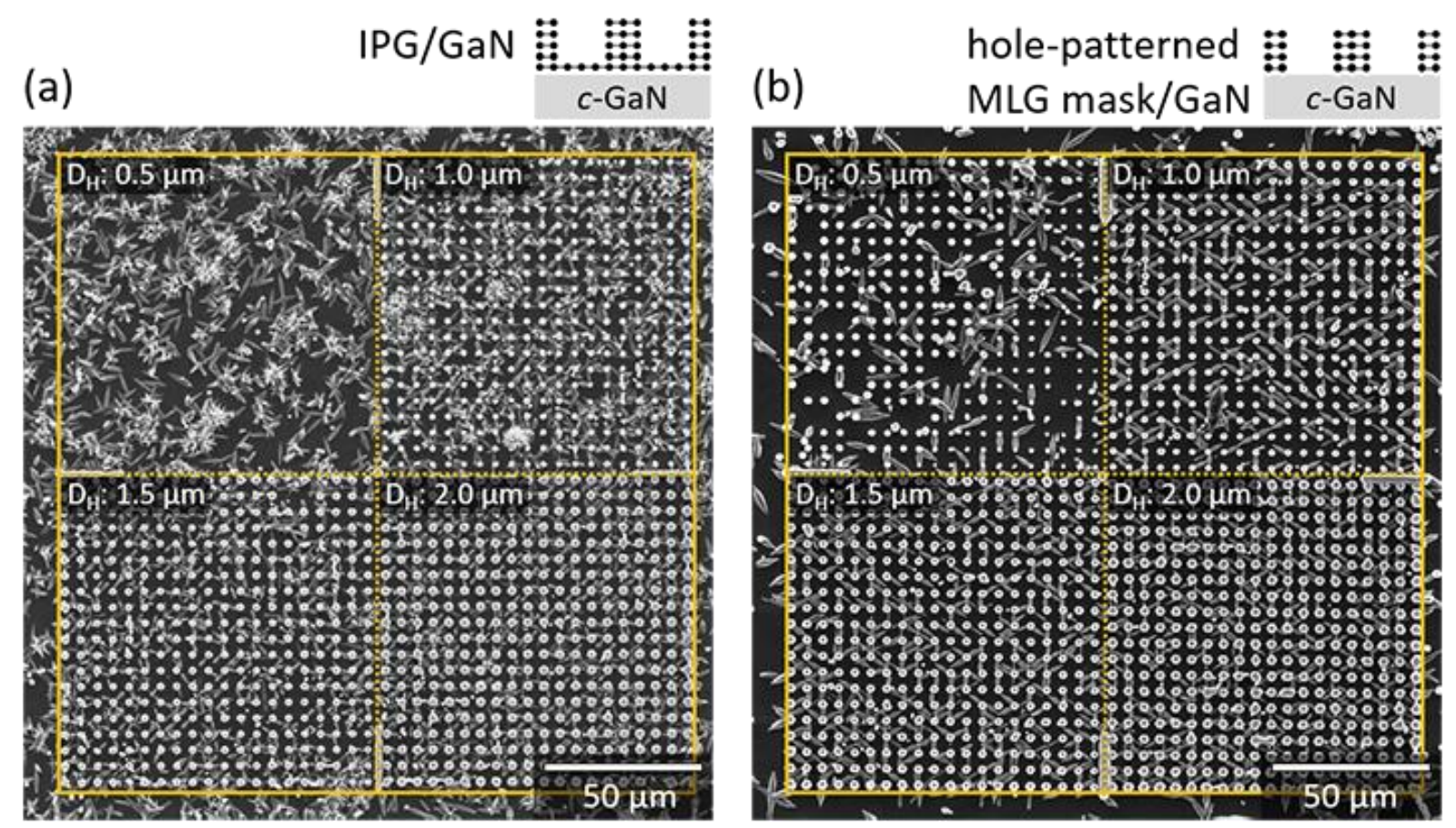

Figure S2. SEM images of ZnO MR arrays grown selectively on (a) IPG-coated $c-G a N$ and (b) hole-patterned MLG mask/c-GaN substrates having hole patterns with diverse hole diameters $\left(D_{H}\right)$ from 0.5 to $2.0 \mu \mathrm{m}$. The growth regimes of the sample (a) and (b) correspond to the remote epitaxy and covalent epitaxy, respectively. The spacing of hole opening was fixed to be $5 \mu \mathrm{m}$ for all patterns. 


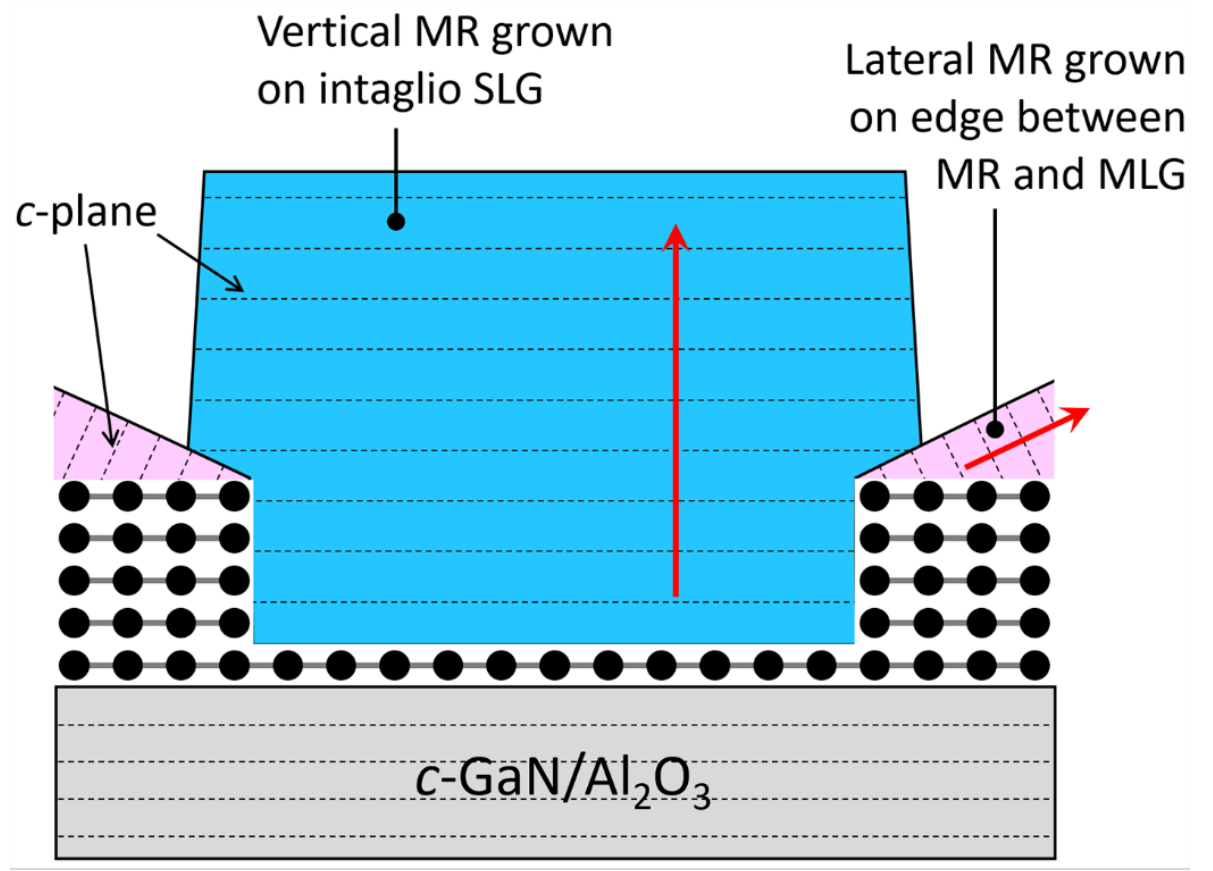

Figure S3. Cross-sectional schematic of vertical MR grown on intaglio SLG area and the lateral MRs grown on edge between remote epitaxial MR and MLG plateau. The dot lines in MRs and substrate indicate the $c$-plane orientation, and the red arrows represent the growth direction of MRs in the [0001]-direction. 

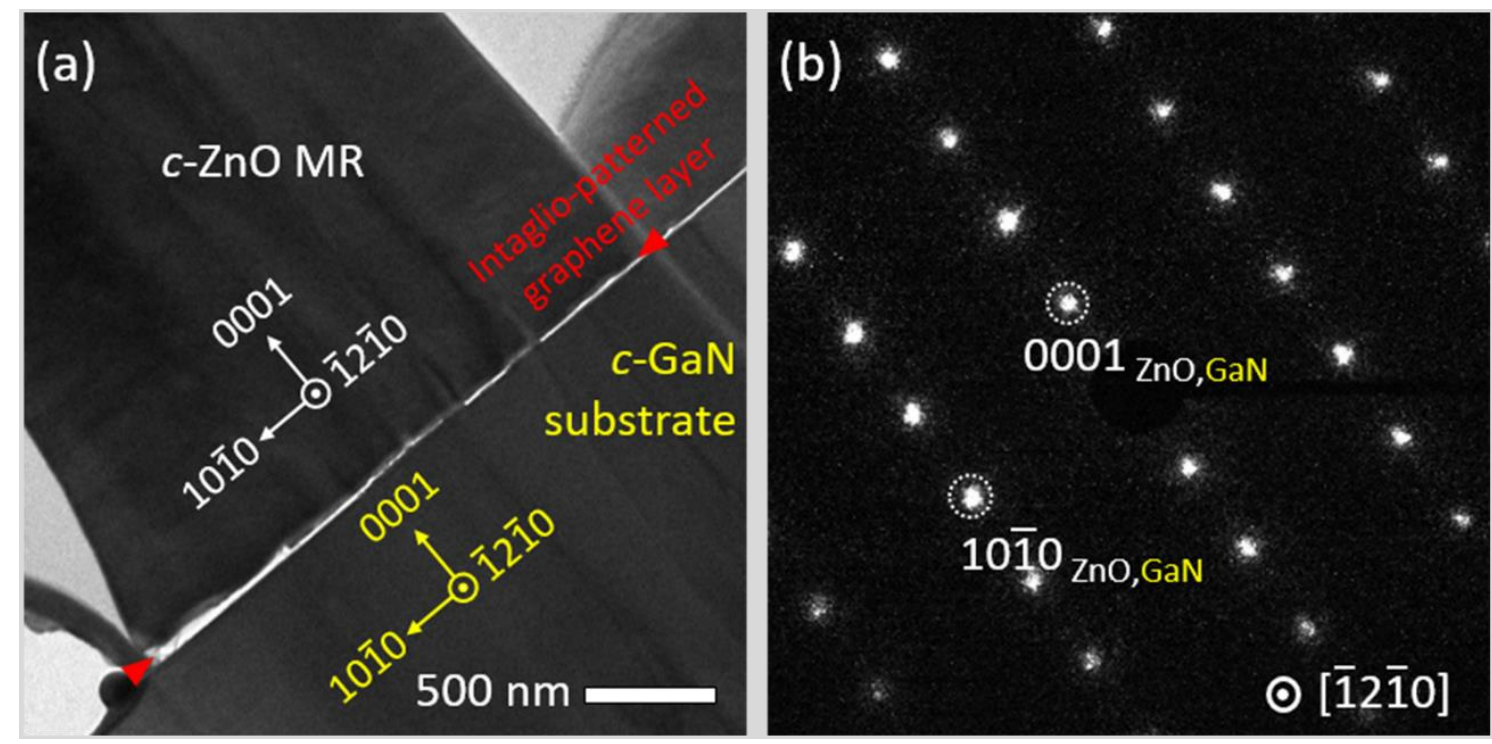

Figure S4. Cross-sectional transmission electron microscopy (TEM) and selected-area electron diffraction (SAED) analyses of c-ZnO/intaglio-patterned graphene/c-GaN heterostructure. (a) Low-magnification scanning-TEM image and (b) SAED pattern of the remote heteroepitaxial c-ZnO MR/patterned graphene/c-GaN substrate obtained from (a). 
(a) Spacing: $4 \mu \mathrm{m}$

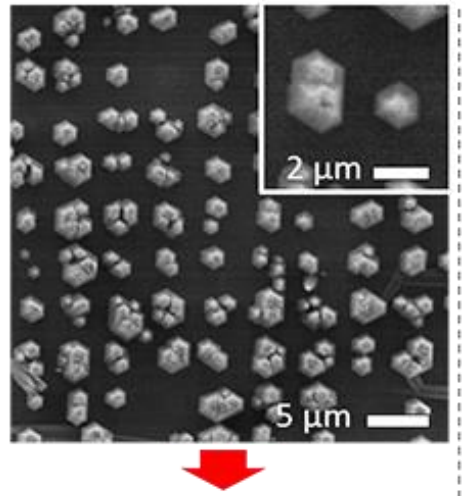

(b) Spacing: $8 \mu \mathrm{m}$

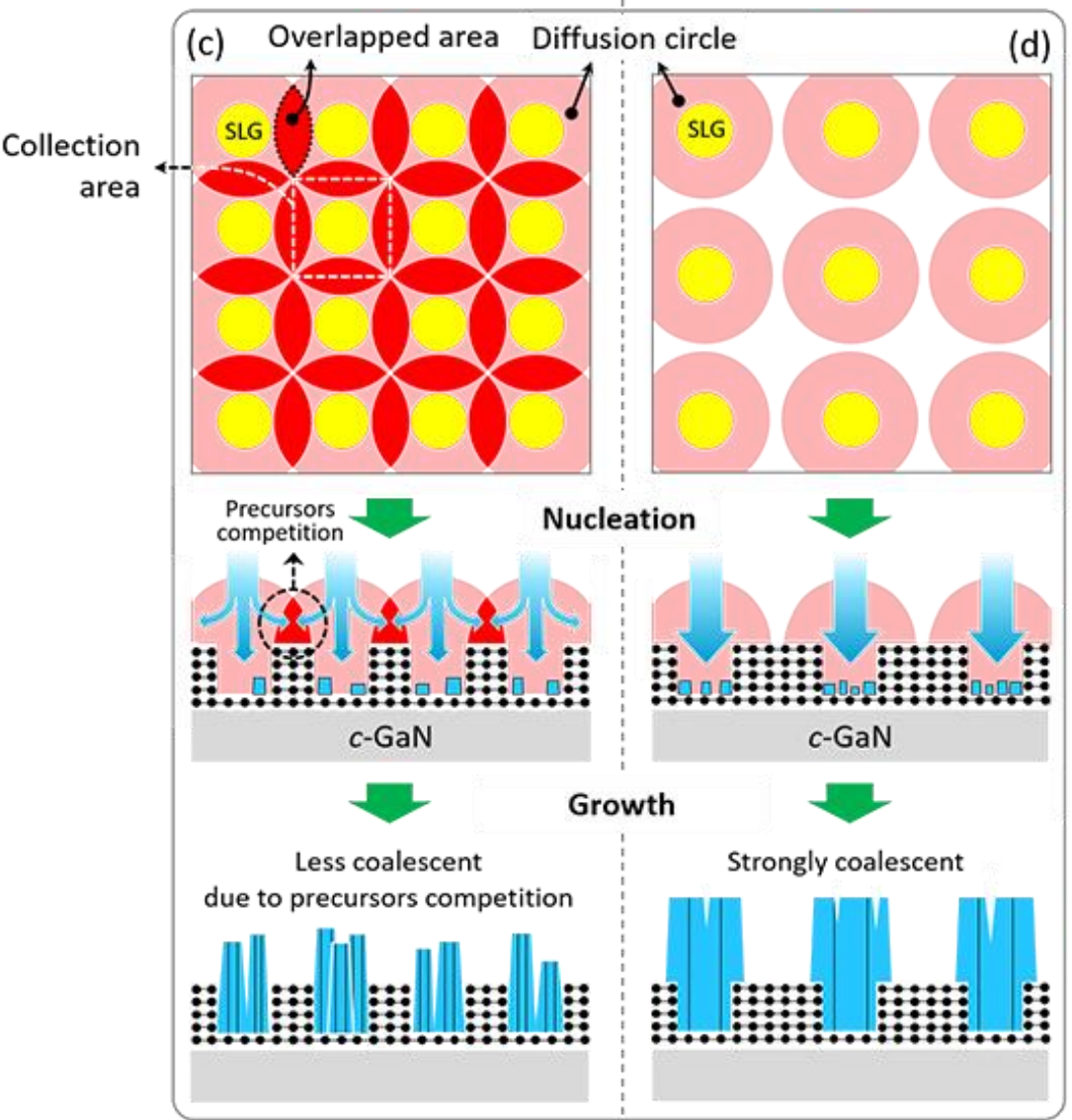

Figure S5. Influence of hole spacing on the nucleation-growth of ZnO MR arrays. Top-view SEM images of MRs grown at spacing of (a) $4 \mu \mathrm{m}$ and (b) $8 \mu \mathrm{m}$. (c, d) Schematic illustrations depicting how the nucleation density and growth rate are strongly governed by the pattern spacing in terms of competition of precursor sharing and collection area. The denser arrays have smaller collection area that supplies lesser amount of precursor. 


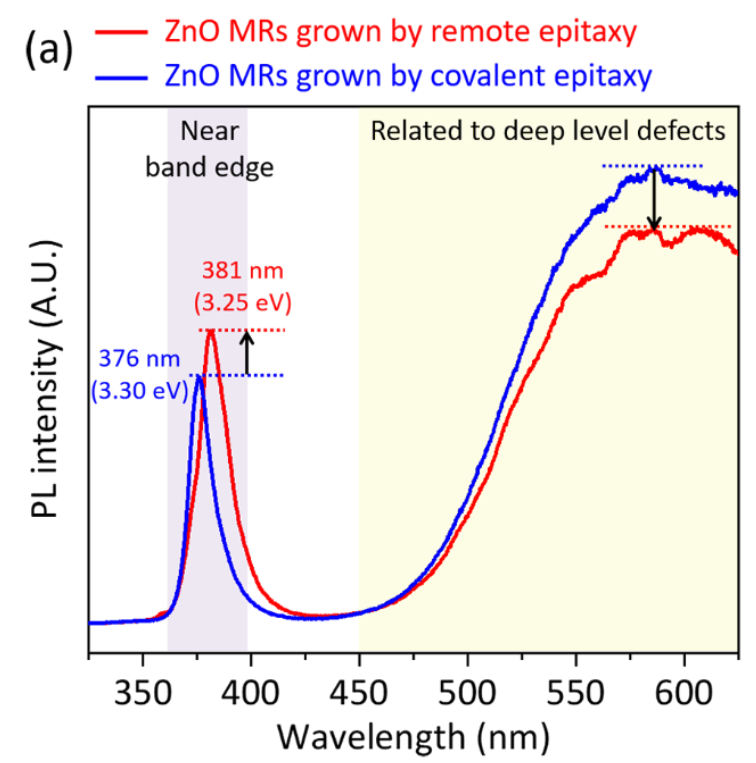

(b)

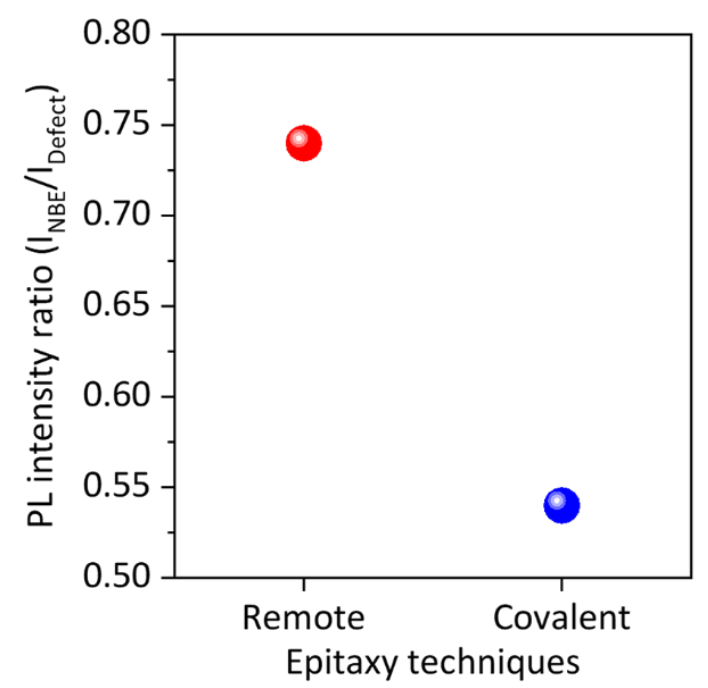

Figure S6. (a) Room-temperature PL spectra of site-selective ZnO MRs grown by covalent epitaxy (blue line) and remote epitaxy (red line). (b) PL intensity ratio of NBE peak-to-defect level band of ZnO MR grown by remote epitaxy (red sphere) and covalent epitaxy (blue sphere). 

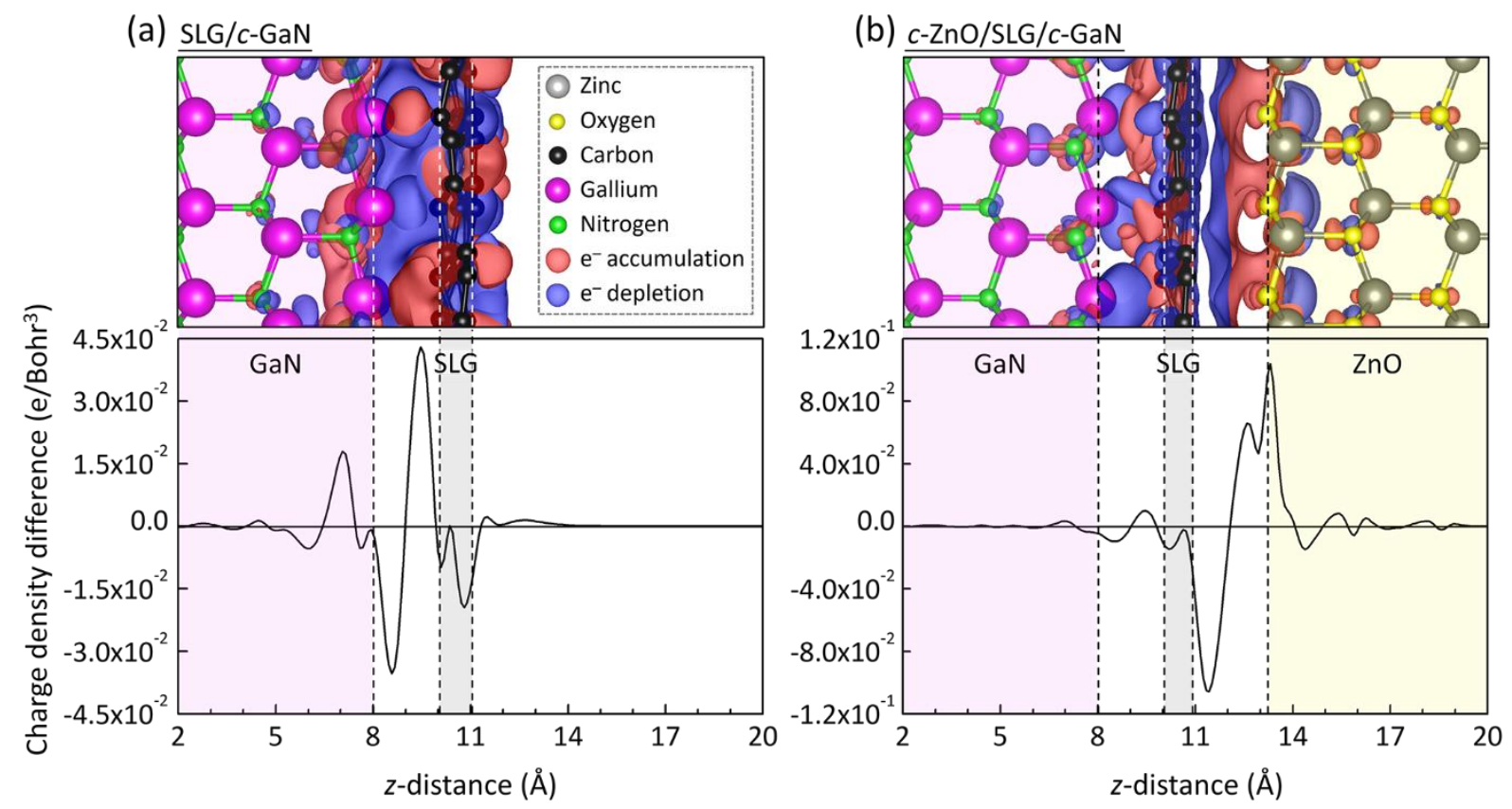

Figure S7. Atomic configurations with charge density isosurfaces (upper panels) and planeaveraged charge density profiles (bottom panels) simulated by density functional theory (DFT) calculation for (a) SLG-coated c-GaN substrate and (b) remote heteroepitaxial c-ZnO/SLG/cGaN structures. The isosurface is contoured at $\pm 0.0005 \mathrm{e} \mathrm{Bohr}^{-3}$. The red and blue isosurfaces signify electron accumulation and depletion, respectively. The dashed lines represent the borderlines of $c$-GaN, SLG, and c-ZnO. 